\title{
On chromatic number and minimum cut
}

\section{Alishahi, Meysam; Hajiabolhassan, Hossein}

Published in:

Journal of Combinatorial Theory. Series B

Link to article, DOI:

10.1016/j.jctb.2019.02.007

Publication date:

2019

Document Version

Peer reviewed version

Link back to DTU Orbit

Citation (APA):

Alishahi, M., \& Hajiabolhassan, H. (2019). On chromatic number and minimum cut. Journal of Combinatorial Theory. Series B, 139, 27-46. https://doi.org/10.1016/j.jctb.2019.02.007

\section{General rights}

Copyright and moral rights for the publications made accessible in the public portal are retained by the authors and/or other copyright owners and it is a condition of accessing publications that users recognise and abide by the legal requirements associated with these rights.

- Users may download and print one copy of any publication from the public portal for the purpose of private study or research.

- You may not further distribute the material or use it for any profit-making activity or commercial gain

- You may freely distribute the URL identifying the publication in the public portal

If you believe that this document breaches copyright please contact us providing details, and we will remove access to the work immediately and investigate your claim 


\title{
On chromatic number and minimum cut
}

\author{
Meysam Alishahi ${ }^{\mathrm{a}}$, Hossein Hajiabolhassan ${ }^{\text {b,c }}$ \\ a Faculty of Mathematical Sciences, Shahrood University of Technology, \\ Shahrood, Iran \\ b Department of Applied Mathematics and Computer Science, Technical University \\ of Denmark, DK-2800 Lyngby, Denmark \\ c Department of Mathematical Sciences, Shahid Beheshti University, P.O. Box \\ 19839-69411, Tehran, Iran
}

\section{A R T I C L E I N F O}

\section{Article history:}

Received 9 March 2017

Available online $\mathrm{xxxx}$

\section{Keywords:}

Chromatic number

Tree Kneser graph

Minimum cut

Alternating partial 2-coloring
A B S T R A C T

For a graph $G$, the tree Kneser graph $\operatorname{KG}\left(G, \mathcal{T}_{t}\right)$ has all tree subgraphs of $G$ with $t$ vertices as vertex set and two vertices are adjacent if their corresponding trees are edge-disjoint. Also, the $r$-th cut number of $G$ is the minimum number of edges between the parts of a partition of the vertex set of $G$ into two parts such that each part has size at least $r$. In this paper, we investigate the chromatic number of tree Kneser graphs. Roughly speaking, we prove that for any nonnegative integer function $t=t(n)$, where $n-t=o(n)$, if $n$ is sufficiently large, then for any dense graph $G$ with $n$ vertices, the chromatic number of the tree Kneser graph $\operatorname{KG}\left(G, \mathcal{T}_{t}\right)$ is equal to the $(n-t+1)$-th cut number of $G$. In particular, as a consequence of this result, if $n$ is large enough, then for any dense graph $G$ with $n$ vertices, the chromatic number of the tree Kneser graph $\operatorname{KG}\left(G, \mathcal{T}_{n}\right)$ is equal to the minimum cut (minimum degree) of $G$. This result can be somehow considered a reminiscent of the Nash-Williams disjoint trees theorem. The proof is based on a lower bound for the chromatic number of graphs found by the present authors Alishahi and Hajiabolhassan (2015) [1] which is inspired by Tucker's lemma, an equivalent combinatorial version of the Borsuk-Ulam theorem. Finally, motivated by the aforementioned results, we close the

E-mail addresses: meysam_alishahi@shahroodut.ac.ir (M. Alishahi), hhaji@sbu.ac.ir (H. Hajiabolhassan). 
paper by a discussion on the complexity of determining the $r$-th cut number of graphs.

(C) 2019 Elsevier Inc. All rights reserved.

\section{Introduction}

Throughout the paper, the symbol $[n]$ stands for the set $\{1, \ldots, n\}$. The Kneser graph $\mathrm{KG}(n, k)$ is a graph whose vertex set consists of all $k$-subsets of $[n]$ and two vertices are adjacent if their corresponding $k$-subsets are disjoint. In 1955, Kneser [10] posed a conjecture about the chromatic number of $\operatorname{KG}(n, k)$. He proved that $\operatorname{KG}(n, k)$ can be properly colored by $n-2 k+2$ colors and conjectured that this number of colors is the least possible. In a breakthrough paper, Lovász [13] proved this conjecture with the help of algebraic topology. Actually, Lovász presented a general lower bound for the chromatic number of graphs which is tight in the case of Kneser graphs $\operatorname{KG}(n, k)$.

Throughout the paper, by a nonempty graph, we mean a graph with nonempty edge set. For a graph $G$ and a nonempty family $\mathcal{F}$ of nonempty graphs, the general Kneser graph $\operatorname{KG}(G, \mathcal{F})$ is a graph whose vertex set consists of all subgraphs of $G$ isomorphic to some members of $\mathcal{F}$ and two vertices are adjacent if their corresponding subgraphs are edge-disjoint. When $\mathcal{F}=\{H\}$, we would prefer to use $\operatorname{KG}(G, H)$ instead of $\operatorname{KG}(G, \mathcal{F})$. Several well-known families of graphs can be represented in the form of general Kneser graphs. For instance, the general Kneser graphs $\operatorname{KG}\left(n K_{2}, k K_{2}\right) \cong \operatorname{KG}\left(K_{1, n}, K_{1, k}\right)$, $\mathrm{KG}\left(C_{n}, k K_{2}\right)$, and $\operatorname{KG}\left(C_{n}, P_{d+1}\right)$, are respectively isomorphic to the Kneser graph $\mathrm{KG}(n, k)$, the Schrijver graph $\mathrm{SG}(n, k)$ and the circular complete graph $K_{\frac{n}{d}}$, where $P_{d+1}$ is a path with $d+1$ vertices.

\subsection{Alternating partial 2-coloring}

In this subsection, unless otherwise stated, we shall consider graphs which are not necessarily simple. For a graph $G$ and its subgraph $H$, throughout the paper, we write $G-E(H)$ and $G-H$ for the subgraphs of $G$ obtained by removing the edges and the vertices of $H$, respectively. Let $G$ be a graph and $\sigma: e_{1}<\cdots<e_{|E(G)|}$ be a linear ordering of $E(G)$. A partial 2-coloring of $E(G)$ is an assignment of two colors red and blue to some edges of $G$. Note that some edges might receive no color. For an edge $e \in E(G)$, if the red (resp. blue) color is assigned to $e$, then we call this edge a red edge (resp. blue edge) and if no color is assigned to $e$, then we call it a neutral edge. Moreover, the red subgraph $G^{R}$ (resp. blue subgraph $G^{B}$ ) is a spanning subgraph of $G$ whose edge set is the set of all red (resp. blue) edges of $G$. A partial 2-coloring is called $\sigma$-alternating if either it has no colored edge or any two consecutive (respect to ordering $\sigma$ ) colored edges of it receive different colors, i.e., if $e_{i_{1}}, \ldots, e_{i_{k}}$ are the colored edges, where $i_{1}<\ldots<i_{k}$, then 
for each $j \in[k-1]$, the edges $e_{i_{j}}$ and $e_{i_{j+1}}$ have different colors. If there is no ambiguity in the ordering $\sigma$, then such a coloring is simply called an alternating partial 2-coloring. The length of a $\sigma$-alternating partial 2-coloring of $E(G)$ is the number of colored edges, i.e., $\left|E\left(G^{R}\right)\right|+\left|E\left(G^{B}\right)\right|$. Note that, in view of the definition of $\sigma$-alternating, for any $\sigma$-alternating partial 2-coloring, we have $\left\|E\left(G^{R}\right)|-| E\left(G^{B}\right)\right\| \leq 1$. For a subgraph $H$ of $G$, the set of neutral edges of $E(H)$ is denoted by $\operatorname{NEU}(H)$. Moreover, the set of neutral edges of $E(H)$ incident with a vertex $v$ of $H$ is denoted by $\operatorname{NEU}(v, H)$. Using the topological tools, the present authors proved the following theorem.

Theorem A. [1,2] Let $G$ be a nonempty graph and $\mathcal{F}$ be a nonempty family containing some subgraphs of $G$. Also, let $\sigma: e_{1}<\cdots<e_{|E(G)|}$ be a linear ordering. If for any $\sigma$-alternating partial 2-coloring of $E(G)$ of length $k$, at least one of $G^{R}$ and $G^{B}$ contains a subgraph isomorphic to some member of $\mathcal{F}$, then

$$
\chi(\operatorname{KG}(G, \mathcal{F})) \geq|E(G)|-k+1
$$

It should be mentioned that the lower bound presented in Theorem A is originally proved in [1] stated in a different language.

\subsection{The $i$-th cut number}

Let $G$ be a graph with $n$ vertices. For two disjoint nonempty subsets $A, B$ of $V(G)$, by $E(A, B)$, we mean the set of edges in $E(G)$ with one end in $A$ and the other end in $B$. Let $i$ be an integer, where $1 \leq i \leq \frac{n}{2}$. A partition $(X, Y)$ of $V(G)$ is called an $i$-cut of $G$ if $\min \{|X|,|Y|\} \geq i$. For any integer $1 \leq i \leq \frac{n}{2}$, define the $i$-th cut number of a graph $G$, denoted $\operatorname{cut}_{i}(G)$, to be the minimum of $|E(X, Y)|$, where the minimum is taken over all $i$-cuts $(X, Y)$ of $G$, i.e.,

$$
\operatorname{cut}_{i}(G)=\min \{|E(S, V(G) \backslash S)|: S \subseteq V(G), \quad|S| \geq i,|V(G) \backslash S| \geq i\}
$$

To clarify the definition, we compute the $i$-th cut numbers of $K_{n}$ and $K_{n, n}$. Let $(S, T)$ be an $i$-cut of $K_{n}$, where $|S| \leq|T|$ and $1 \leq i \leq \frac{n}{2}$. Clearly, $|E(S, T)|=s(n-s)$, where $s=|S|$. Since $f(s)=s(n-s)$ is increasing on $\left[i, \frac{n}{2}\right]$, it takes its minimum in $s=i$. Accordingly, $\operatorname{cut}_{i}\left(K_{n}\right)=i(n-i)$ for each $1 \leq i \leq \frac{n}{2}$. Now, let $K_{n, n}=(X, Y)$ and $(S, T)$ be an $i$-cut of $K_{n, n}$, where $|S| \leq|T|$ and $1 \leq i \leq n$. For $x=|S \cap X|$ and $y=|S \cap Y|$, we have $|E(S, T)|=x(n-y)+y(n-x)$. To compute cut ${ }_{i}\left(K_{n, n}\right)$, we need to find the minimum of the function $g(x, y)=(x+y) n-2 x y$ over $\{(x, y): x, y \in\{0, \ldots, n\}, i \leq x+y \leq n\}$. It is not hard to see that the minimum is $n i-2\left\lfloor\frac{i}{2}\right\rfloor\left\lceil\frac{i}{2}\right\rceil$ taking place when $x=\left\lceil\frac{i}{2}\right\rceil$ and $y=\left\lfloor\frac{i}{2}\right\rfloor$. Therefore, $\operatorname{cut}_{i}\left(K_{n, n}\right)=n i-2\left\lfloor\frac{i}{2}\right\rfloor\left\lceil\frac{i}{2}\right\rceil$ for each $1 \leq i \leq n$. Note that $\operatorname{cut}_{1}(G)$ is a well-known parameter known as the minimum cut of $G$, denoted by $\operatorname{cut}(G)$.

Also, for a partition $P=\left(V_{1}, \ldots, V_{m}\right)$ of $V(G)$, any edge of $G$ whose ends are in different parts of $P$ is called a cross-edge of $P$. Tutte [15] and Nash-Williams [14] independently proved that a graph $G$ contains $k$ pairwise edge-disjoint spanning trees if 
and only if for any partition $P=\left(V_{1}, \ldots, V_{m}\right)$ of $V(G)$, there are at least $k(m-1)$ cross-edges of $P$. For $n \geq 2$, let $\mathcal{T}_{n}$ be the family of all pairwise non-isomorphic trees with $n$ vertices. Clearly, the aforementioned result by Tutte and Nash-Williams can be restated as follows:

For any graph $G$ with $n$ vertices, $\omega\left(\operatorname{KG}\left(G, \mathcal{T}_{n}\right)\right) \geq k$ if and only if there are at least $k(m-1)$ cross-edges of any partition $P=\left(V_{1}, \ldots, V_{m}\right)$ of $V(G)$.

In other words, it shows that the clique number of $\operatorname{KG}\left(G, \mathcal{T}_{n}\right)$ is the floor of the minimum possible ratio of the number of cross-edges of a partition $P=\left(V_{1}, \ldots, V_{m}\right)$ to $m-1$, where the minimum is taken over all partitions $P=\left(V_{1}, \ldots, V_{m}\right)$ of $V(G)$ with $m>1$. Accordingly, this value is at least $\left[\frac{\operatorname{cut}_{1}(G)-1}{2}\right]$, see [11]. Moreover, for an integer $r$, where $0 \leq r \leq \frac{n}{2}-1$, let $(X, Y)$ be an $(r+1)$-cut of $G$ with $E(X, Y)=\left\{e_{1}, \ldots, e_{C}\right\}$. For any vertex $T$ of $\operatorname{KG}\left(G, \mathcal{T}_{n-r}\right)$, define $c(T)$ to be the minimum $i$ such that $e_{i} \in E(T)$. One can check that $c$ is a proper $C$-coloring of $\operatorname{KG}\left(G, \mathcal{T}_{n-r}\right)$. Hence, we have

$$
\chi\left(\mathrm{KG}\left(G, \mathcal{T}_{n-r}\right)\right) \leq \operatorname{cut}_{r+1}(G) .
$$

Since the clique number of a graph can be considered as an estimation of its chromatic number, the prior discussion implies that

$$
\left\lceil\frac{\operatorname{cut}_{1}(G)-1}{2}\right\rceil \leq \chi\left(\operatorname{KG}\left(G, \mathcal{T}_{n}\right)\right) \leq \operatorname{cut}_{1}(G) .
$$

Note that we have found an upper bound for $\chi\left(\operatorname{KG}\left(G, \mathcal{T}_{n-r}\right)\right)$ in terms of $\operatorname{cut}_{r+1}(G)$ while the lower bound has been proved just for $r=0$. Although it is valuable to have a similar lower bound in terms of $\operatorname{cut}_{r+1}(G)$ for the chromatic number of $\operatorname{KG}\left(G, \mathcal{T}_{n-r}\right)$, to prove it, in our approach we need an appropriate generalization of Tutte and Nash-Williams theorem providing a sufficient condition for the existence of $k$ pairwise edge-disjoint subtrees of $G$ with $n-r$ vertices. As far as we know, no one has proved such a generalization though it is a nice question to investigate.

Motivated by the preceding discussion, it is interesting to investigate the chromatic number of $\operatorname{KG}\left(G, \mathcal{T}_{n-r}\right)$ for $0 \leq r \leq \frac{n}{2}-1$. In this regard, the present authors (see Theorem 2 in [2]) proved the following theorem which is originally stated in terms of Turán number.

Theorem B. [2] Let $t \in\{0, \ldots, n-2\}$ be an integer and $G$ be a nonempty multi-graph with $n$ vertices such that each of its edges has the multiplicity at least 2 . The chromatic number of $\operatorname{KG}\left(G, \mathcal{T}_{t}\right)$ is equal to $m(G, t)$, that is, the minimum number of edges should be removed from $G$ so that any connected component of the resulting graph has at most $t-1$ vertices.

One can see that this result implies the following statement; 
If $G$ is a nonempty connected multi-graph, each of its edges has the multiplicity at least 2 , then $\chi\left(\operatorname{KG}\left(G, \mathcal{T}_{n-r}\right)\right)=\operatorname{cut}_{r+1}(G)$ for each $r \in$ $\left\{0, \ldots,\left\lceil\frac{|V(G)|}{3}\right\rceil-1\right\}$.

Clearly, to prove the assertion, it suffices to show that $m(G, n-r)=\operatorname{cut}_{r+1}(G)$ for each $r \in\left\{0, \ldots,\left[\frac{|V(G)|}{3}\right]-1\right\}$. In view of the definition of $\operatorname{cut}_{r+1}(G)$, it is clear that $m(G, n-r) \leq \operatorname{cut}_{r+1}(G)$. Now, consider $F \subseteq E(G)$ with $|F|=m(G, n-r)$ and such that any component of $G-F$ has at most $n-r-1$ vertices. Also, let $Q_{1}, \ldots, Q_{m}$ be the components of $G-F$, where $\left|Q_{1}\right| \leq \cdots \leq\left|Q_{m}\right|$. Consider the smallest $l \in[m]$ such that $\left|Q_{1}\right|+\cdots+\left|Q_{l}\right| \geq r+1$. First note that $l<m$. We claim that $\left|Q_{1}\right|+\cdots+\left|Q_{l}\right| \leq n-r-1$. On the contrary, suppose that $\left|Q_{1}\right|+\cdots+\left|Q_{l}\right| \geq n-r$. Due to the minimality of $l$, we clearly have $\left|Q_{1}\right|+\cdots+\left|Q_{l-1}\right| \leq r$, implying that $\left|Q_{l}\right| \geq n-2 r$. On the other hand, we have

$$
n-2 r \leq\left|Q_{l}\right| \leq\left|Q_{l+1}\right| \leq n-\left|\bigcup_{i=1}^{l} Q_{l}\right| \leq n-(n-r)=r
$$

concluding that $3 r \geq n$ which is a contradiction. Now, set $S=\bigcup_{i=1}^{l} Q_{i}$. In view of the definition of $S$ and since $r+1 \leq|S| \leq n-r-1$, we have

$$
\operatorname{cut}_{r+1}(G) \leq|E(S, V(G) \backslash S)| \leq m(G, n-r),
$$

completing the proof.

\subsection{Main results}

Unfortunately, the idea used in the proof of Theorem B does not work when we deal with the simple graphs. Hereafter, unless otherwise stated, we consider only simple graphs and by a graph we mean a simple graph. The next theorem is the main result of this paper which presents some sufficient conditions for an $n$-vertex simple graph $G$ to satisfy the equation $\chi\left(\operatorname{KG}\left(G, \mathcal{T}_{n-r}\right)\right)=\operatorname{cut}_{r+1}(G)$.

Theorem 1. Let $\delta$ be a real number and $r=r(n)$ be a nonnegative integer function, where $\frac{5}{6}<\delta<1$ and $r=o(n)$. There is a constant $N(\delta, r)$ such that if $n \geq N(\delta, r)$, then for any graph $G$ with $n$ vertices and $\delta(G) \geq \delta n$, we have

$$
\chi\left(\operatorname{KG}\left(G, \mathcal{T}_{n-r}\right)\right)=\operatorname{cut}_{r+1}(G) .
$$

One can simply see that for $n \geq 6$, the cut number of a graph $G$, satisfying the conditions of Theorem 1 , is $\delta(G)$. In view of this observation, we shall prove the next theorem. 
Theorem 2. Let $n$ be a positive integer and $\delta$ be a real number, where $\frac{5}{6}<\delta<1$ and $n \geq$ $\max \left\{4716, \frac{828}{6 \delta-5}\right\}$. If $G$ is a graph with $n$ vertices and $\delta(G) \geq \delta n$, then $\chi\left(\operatorname{KG}\left(G, \mathcal{T}_{n}\right)\right)=$ $\delta(G)$.

By the aforementioned results, one can introduce some graphs whose chromatic numbers are about twice of their clique numbers. To see this, note that if $n \geq 4716$, then $\chi\left(\operatorname{KG}\left(K_{n}, \mathcal{T}_{n}\right)\right)=n-1$. Also, if $t$ is an even integer, then the complete graph $K_{t}$ is decomposable into edge-disjoint Hamiltonian paths. Hence, for any integer $n \geq 2$, the clique number of $\operatorname{KG}\left(K_{n}, \mathcal{T}_{n}\right)$ is $\left\lfloor\frac{n}{2}\right\rfloor$, while for $n \geq 4716$, we have $\chi\left(\operatorname{KG}\left(K_{n}, \mathcal{T}_{n}\right)\right)=n-1$.

It would be of interest to know if we can drop some conditions from the hypothesis of Theorem 1. Simply, one can see that Theorem 1 does not hold for sparse graphs. To see this, consider the tree Kneser graph $\operatorname{KG}\left(G, \mathcal{T}_{n}\right)$, where $G$ is a connected graph with $n \geq 6$ vertices and $|E(G)| \leq 2 n-3$. Clearly, the graph $\operatorname{KG}\left(G, \mathcal{T}_{n}\right)$ has no edge, and consequently, its chromatic number is equal to 1 , while the cut number of $G$ can be equal to 3 .

In addition, it should be noticed that for each $r \geq\left\lceil\frac{|V(G)|}{3}\right\rceil$, not only Theorem 1 , but also the statement right after Theorem B does not hold in general. To see this, let $k$ be an integer, where $k \geq 5$. Set $n=3 k$ and consider the graph $H$ with $n$ vertices, consisting of three disjoint copies of $K_{k}$ and such that for any two of these three copies, there is exactly one edge between them. Also, let $G$ be the multi-graph obtained from $H$, by multiplying each edge of $H$. One can simply check that $\chi\left(\operatorname{KG}\left(H, \mathcal{T}_{n-r}\right)\right) \leq 3$ and $\chi\left(\operatorname{KG}\left(G, \mathcal{T}_{n-r}\right)\right) \leq 6$, while $\operatorname{cut}_{r+1}(H) \geq(k-1) \geq 4$ and $\operatorname{cut}_{r+1}(G) \geq 2(k-1) \geq 8$ for each $r \in\left\{k, \ldots,\left\lfloor\frac{n}{2}\right\rfloor-1\right\}$.

However, it is interesting to extend Theorem 1 by finding some other conditions or improving the current conditions such that we still have the conclusion of this theorem.

\subsection{Plan}

This paper is organized as follows. Section 2 is devoted to the proof of Theorems 1 and 2. In Section 3, we end the paper by a discussion on the complexity of finding the $t$-th cut number of graphs. Roughly speaking, we prove that the problem of finding the $t$-th cut number of graphs is solvable in polynomial time if $t$ is a constant and it is an NP-hard problem even if $t$ and the input graphs satisfy the hypotheses of Theorem 1.

\section{Proofs of Theorem 1 and Theorem 2}

For two graphs $G$ and $H$, an $H$-packing of $G$ is a set $\left\{H_{1}, \ldots, H_{t}\right\}$ of pairwise edgedisjoint subgraphs of $G$ such that for each $1 \leq i \leq t$, the graph $H_{i}$ is isomorphic to $H$. The $H$-packing number of $G$ is the maximum cardinality of an $H$-packing of $G$. An $H$-packing of $G$ is called a monogamous $H$-packing if every pair of vertices of $G$ appears in at most one copy of $H$ in the packing. In [12], it was shown that for positive even integers $m$ and $n$, the complete bipartite graph $K_{m, n}$ has a monogamous $C_{4}$-decomposition 
if and only if $(m, n)=(2,2)$ or $6 \leq n \leq m \leq 2 n-2$. Clearly, any $K_{r}$-packing of any graph $G$ is monogamous. The problem of finding as many as possible vertex-disjoint complete subgraphs isomorphic to $K_{r}$ has been studied in several articles. Hajnal and Szemerédi [7] proved the next theorem.

Theorem C. [7] Let $G$ be a graph with $n$ vertices. If $\delta(G) \geq\left(1-\frac{1}{r}\right) n$, then $G$ contains $\left\lfloor\frac{n}{r}\right\rfloor$ vertex-disjoint copies of $K_{r}$.

Let $G$ be a graph and $\sigma: e_{1}<\cdots<e_{|E(G)|}$ be a linear ordering of $E(G)$. For a vertex $v \in V(G)$, a path $u v w$ is celled a $(\sigma, v)$-consecutive path if the edges $u v$ and $v w$ are consecutive in the ordering $\sigma$. Since in any $\sigma$-alternating partial 2-coloring of $E(G)$, we do not assign the same color to the edges of any $(\sigma, v)$-consecutive path, we have the following observation.

Lemma 1. Let $G$ be a graph, $v \in V(G)$, and $\sigma$ be a linear ordering of $E(G)$. Consider a $\sigma$-alternating partial 2-coloring of $E(G)$ and let $E_{v}^{R}$ be a subset of $E(G)$ which contains all red edges incident to $v$. Moreover, suppose that there are $t_{1}$ edge-disjoint $(\sigma, v)$-consecutive paths such that $t_{2}$ number of them intersect $E_{v}^{R}$. Then there are at least $t_{1}-t_{2}$ neutral edges incident with $v$ in $E(G) \backslash E_{v}^{R}$.

Now, we are ready for the proof of Theorem 1.

\subsection{Proof of Theorem 1}

In view of Inequality 1, to fulfill Theorem 1, it suffices to show that

$$
\chi\left(\mathrm{KG}\left(G, \mathcal{T}_{n-r}\right)\right) \geq \operatorname{cut}_{r+1}(G) .
$$

Therefore, using Theorem A, we shall have proved Theorem 1, if we prove the next lemma.

Lemma 2. Let $\delta$ be a real number and $r=r(n)$ be a nonnegative integer function, where $\frac{5}{6}<\delta<1$ and $r=o(n)$. There is a constant $N(\delta, r)$ such that if $n \geq N(\delta, r)$, then for any graph $G$ with $n$ vertices and $\delta(G) \geq \delta$, there is an ordering $\sigma: e_{1}<\cdots<e_{|E(G)|}$ such that for any $\sigma$-alternating partial 2-coloring of $E(G)$ with length $|E(G)|-\operatorname{cut}_{r+1}(G)+1$, at least one of $G^{R}$ and $G^{B}$ has a connected component with at least $n-r$ vertices.

It is worth mentioning that although there are some other topological lower bounds for the chromatic number of general Kneser graphs, Theorem A has a privilege since it can be seen as a pure combinatorial tool (even if it also relies on a topological proof) for finding a lower bound for the chromatic number of general Kneser graphs. For using the power of Theorem A, one needs to propose an appropriate ordering and somehow prove a Turán type result with respect to the ordering. This approach can be employed by any 
one even with no background in topological combinatorics. The proof of Lemma 2 can be considered as an example of such application which is divided into two main parts. In the first part, we will define an ordering $\sigma$ while in the second part, we will prove that the ordering $\sigma$ satisfies the assertion of Lemma 2.

Proof of Lemma 2. Assume that $n \geq N$, where $N=N(\delta, r)$ is a constant which will be determined during the proof. Let $G$ be a graph with $n \geq N$ vertices and $\delta(G) \geq \delta n$.

Part (I): Definition of the ordering $\sigma$.

Let $V(G)=\left\{v_{1}, v_{2}, \ldots, v_{n}\right\}$. Set $l=\left\lceil\frac{26(r+1)}{\delta}\right\rceil=o(n)$ and let $n=4 a+b$, where $a$ and $b$ are positive integers and $1 \leq b \leq 4$. Join $4-b$ new vertices to $G$ in order to obtain the graph $G^{\prime}=G \vee K_{4-b}$. Also, let $F$ be a graph with exactly $a+1$ connected components each isomorphic to $K_{4}$. By Theorem $\mathrm{C}$, there are at least $\frac{1}{36} n$ edge-disjoint copies of $F$ in $G^{\prime}$. Let $N$ be large enough such that $\frac{1}{36} n \geq 4 l+3$. Let $\left\{F_{1}, F_{2}, \ldots, F_{4 l+3}\right\}$ be $4 l+3$ edge-disjoint copies of $F$ in $G^{\prime}$. For each $F_{i}$ and for each connected component of $F_{i}$ which is a subgraph of $G$, choose a four cycle $C_{4}$ of this component. Clearly, these $C_{4}$ 's form a monogamous $C_{4}$-packing of $G$. Call each $C_{4}$ of this packing a 4 -block. Also, one can see that every vertex of $G$ appears in at least $4 l$ and at most $4 l+3$ number of 4-blocks.

Construct a bipartite graph with two parts $W$ and $W^{\prime}$, where $W$ is a set consisting of $l$ copies of each vertex of $G$ and $W^{\prime}$ is the set of all 4 -blocks. For each vertex $v \in V(G)$, join each of $l$ copies of $v$ to any 4-block in $W^{\prime}$ containing $v$. One can check that the degree of every vertex in the part $W$ is at least $4 l$ and also the degree of every vertex in the part $W^{\prime}$ is exactly $4 l$. In view of Hall's Theorem, this bipartite graph has a matching saturating all vertices in $W$. For any vertex $v_{i} \in V(G)$, set $A_{i}$ to be the set of 4-blocks assigned to $l$ copies of $v_{i}$ through the aforementioned matching. Note that $\left|A_{i}\right|=l$ and since 4-blocks form a monogamous $C_{4}$-packing, the intersection of any two 4-blocks in $A_{i}$ is $v_{i}$. For each $i \in[n]$, define $H_{i}$ to be the subgraph of $G$ consisting of all 4-blocks in $A_{i}$. In other words, $H_{i}$ is the union of 4-blocks in $A_{i}$. Clearly, $H_{1}, H_{2}, \ldots, H_{n}$ are pairwise edge-disjoint subgraphs of $G$ and for each $i \in[n]$, we have $v_{i} \in V\left(H_{i}\right)$. Also, every vertex of $V\left(H_{i}\right) \backslash\left\{v_{i}\right\}$ has degree 2 in $H_{i}$. Since any vertex of $G$ appears in at most $4 l+3$ numbers of 4 -blocks, the degree of every vertex of the graph $G-\bigcup_{i=1}^{n} E\left(H_{i}\right)$ is at least $\delta n-8 l-6$. Note that since $8 l+6=o(n)$, we can consider $N$ large enough to have $\delta n-8 l-6 \geq \frac{n}{2}+2$. Now, by the well-known result of Dirac, the graph $G-\bigcup_{i=1}^{n} E\left(H_{i}\right)$ contains two edge-disjoint Hamiltonian cycles $C, C^{\prime}$. Without loss of generality, we can assume that $E(C)=\left\{v_{i} v_{i+1}: 1 \leq i \leq n-1\right\} \cup\left\{v_{1} v_{n}\right\}$. Consider the family $M$ defined as follows:

- If $G$ has no odd degree vertex, then set $M=\varnothing$. 
- Otherwise, let $M$ be a family of vertex-disjoint paths in $C^{\prime}$ such that every odd vertex of $G$ is a leaf (vertex of degree one) of these paths and also any leaf of these paths has an odd degree in $G$. One can simply check that such a family exists.

Let $L$ be the spanning subgraph of $G$ whose edge set is $\left.E(M) \cup E(C) \bigcup_{i=1}^{n} E\left(\biguplus_{i}\right)\right)$. Clearly, we have $y=|E(L)| \leq(4 l+4) n$. Consider a maximum $K_{3}$-packing of $G-E(L)$ and call each of its triangles a 3-block. Let $q$ be the number of these 3-blocks. By a result of Huu Hoi (see page 8 in [9]), there are at least

$$
\frac{\epsilon}{3 \nu^{2}}\left(4 \epsilon-\nu^{2}\right) \frac{(\nu-3)(\nu-4)}{(\nu-1)(\nu-2)}=\frac{\epsilon}{3 \nu(\nu-2)}\left(4 \epsilon-\nu^{2}\right)-O(\nu)
$$

edge-disjoint triangles in a graph with $\nu$ vertices and $\epsilon$ edges, where $\nu \geq 5$. Therefore, the number of 3-blocks is at least

$$
\frac{|E(G)|-y}{3 n(n-2)}\left(4|E(G)|-4 y-n^{2}\right)-O(n)=\frac{|E(G)|}{3 n(n-2)}\left(4|E(G)|-n^{2}\right)-o\left(n^{2}\right)
$$

Let $G^{\prime \prime}$ be the graph obtained from $G-E(L)$ by removing the edges of the aforementioned 3-blocks. Clearly, any vertex of $G^{\prime \prime}$ is an even vertex, and therefore, every connected component of $G^{\prime \prime}$ is an Eulerian graph. Note that the way that we choose the family $M$ grantees this property. Let $Q_{1}, Q_{2}, \ldots, Q_{s}$ be the connected components of $G^{\prime \prime}$.

Also, note that the graph $G-E(M)$ is Eulerian. By the following procedure, we will construct an Eulerian tour for $G-E(M)$ and using it, we will introduce the desired ordering $\sigma$.

For $i \in[n]$, at the $i$-th stage, do the following steps:

- Traverse an Eulerian tour of $H_{i}$ started at $v_{i}$.

- Traverse every untraversed 3 -block containing $v_{i}$ started at $v_{i}$. Any two consecutively traversed 3-blocks in this stage are called consecutive.

- If there is a $j \in[s]$ such that $v_{i} \in Q_{j}$ and the edge set of $Q_{j}$ is still untraversed, then consider an Eulerian tour for $Q_{j}$ starting at $v_{i}$ and traverse it.

- Traverse the edge $v_{i} v_{i+1}$ (the indices are taken modulo $n$ ).

- If $i<n$, then start the $(i+1)$-th stage.

Construct an ordering $\pi$ for $E(G)-E(M)$ such that the edges are ordered corresponding to their ordering in the aforementioned Eulerian tour, i.e., if we traverse the edge $e$ before the edge $e^{\prime}$ in the Eulerian tour, then in the ordering $\pi$, we have $e<e^{\prime}$. Now, let the ordering $\sigma$ be an ordering obtained from $\pi$ by putting the edges of $M$ at the end of the ordering $\pi$ such that any two incident edges in $M$ are consecutive.

Hereafter, during the rest of the proof, by ordering $\sigma$, we mean the resulting ordering mentioned in the preceding discussion. 
Part (II): For any $\sigma$-alternating partial 2-coloring of $E(G)$ with length $|E(G)|$ cut $_{r+1}(G)+1$, at least one of $G^{R}$ and $G^{B}$ has a connected component with at least $n-r$ vertices.

Consider an arbitrary fixed $\sigma$-alternating partial 2-coloring of $E(G)$ with length $|E(G)|-\operatorname{cut}_{r+1}(G)+1$. Since

$$
\operatorname{cut}_{r+1}(G)=\min \left\{\left|E_{G}(S, V(G) \backslash S)\right|: S \subseteq V(G), \quad|S| \geq r+1,|V(G) \backslash S| \geq r+1\right\}
$$

we have

$$
\operatorname{cut}_{r+1}(G) \leq(r+1)(n-r-1)=o\left(n^{2}\right) .
$$

Also, since ||$E\left(G^{R}\right)|-| E\left(G^{B}\right) \| \leq 1$ and the number of neutral edges is $\operatorname{cut}_{r+1}(G)-1$, we have

$$
\min \left\{\left|E\left(G^{R}\right)\right|,\left|E\left(G^{B}\right)\right|\right\} \geq \frac{|E(G)|-\operatorname{cut}_{r+1}(G)}{2}>\frac{5}{24} n^{2}-\frac{1}{2}(r+1)(n-r-1) .
$$

In view of the definition of $\sigma$, for any vertex $u \in V(G)$, the number of red (resp. blue) edges incident with $u$ in $G-E(M)$ is at most $\frac{\operatorname{deg}_{G}(u)-\operatorname{deg}_{M}(u)+2}{2}$. Also, according to the ordering of the edges of $M$ in $\sigma$, at most one edge in $E(M)$ incident with $u$ (if there is such an incident edge) is red (resp. blue). Consequently, for each vertex $u \in V(G)$, we have

$$
\max \left\{\operatorname{deg}_{G^{R}}(u), \operatorname{deg}_{G^{B}}(u)\right\} \leq \frac{\operatorname{deg}_{G}(u)+3}{2}
$$

Note that we shall have established Part (II), if we prove the following claim.

Claim 1. $G^{R}$ or $G^{B}$ has a connected component with at least $n-r$ vertices.

Proof of Claim 1. On the contrary, suppose that every connected component of $G^{R}$ and also of $G^{B}$ has at most $n-r-1$ vertices. Let $\Omega_{R}$ and $\Omega_{B}$ be the largest connected component of $G^{R}$ and $G^{B}$, respectively. Note that we have $\max \left\{\left|V\left(\Omega_{R}\right)\right|,\left|V\left(\Omega_{B}\right)\right|\right\} \leq$ $n-r-1$. Also, in view of Inequality 5 , the number of red edges and the number of blue edges in $G$ are both $O\left(n^{2}\right)$, i.e., $\left|E\left(G^{R}\right)\right|=O\left(n^{2}\right)$ and $\left|E\left(G^{B}\right)\right|=O\left(n^{2}\right)$. Therefore, since $r=o(n)$, if $N$ is large enough, then we should have $\min \left\{\left|V\left(\Omega_{R}\right)\right|,\left|V\left(\Omega_{B}\right)\right|\right\} \geq r+1$. Let $H_{R}$ be the bipartite subgraph of $G$ with two parts $U_{R}$ and $V_{R}$, where $\left\{U_{R}, V_{R}\right\}=$ $\left\{V\left(\Omega_{R}\right), V(G) \backslash V\left(\Omega_{R}\right)\right\}$ and $\left|U_{R}\right| \leq\left|V_{R}\right|$. A vertex of $U_{R}$ is adjacent to a vertex of $V_{R}$ if they are adjacent in $G$. Similarly, the bipartite subgraph $H_{B}$ of $G$ has $U_{B}$ and $V_{B}$ as its part, where $\left\{U_{B}, V_{B}\right\}=\left\{V\left(\Omega_{B}\right), V(G) \backslash V\left(\Omega_{B}\right)\right\}$ and $\left|U_{B}\right| \leq\left|V_{B}\right|$. Set $\alpha=\left|U_{R}\right|$ and $\beta=\left|U_{B}\right|$. In view of the former discussion, note that $r+1 \leq \alpha, \beta \leq \frac{n}{2}$. Consider the bipartite graph $H_{R}$. Since there is no red edge in $E\left(H_{R}\right)=E_{G}\left[U_{R}, V_{R}\right]$, each of red 
edges incident with $u$ is in $E\left(G\left[U_{R}\right]\right)$ for each $u \in U_{R}$. Hence, for any vertex $u \in U_{R}$, at most $\alpha-1$ red edges are adjacent to $v$. Therefore, in view of Inequality 6 , we have

$$
\begin{aligned}
|\operatorname{NEU}(G)| & \geq \sum_{u \in U_{R}}\left(\operatorname{deg}_{G}(u)-\operatorname{deg}_{G\left[U_{r}\right]}(u)-\operatorname{deg}_{G^{B}}(u)\right) \\
& \geq \sum_{u \in U_{R}}\left(\operatorname{deg}_{G}(u)-(\alpha-1)-\operatorname{deg}_{G^{B}}(u)\right) \\
& \geq \sum_{u \in U_{R}}\left(\operatorname{deg}_{G}(u)-(\alpha-1)-\frac{\operatorname{deg}_{G}(u)+3}{2}\right) \\
& \geq \sum_{u \in U_{R}}\left(\frac{\operatorname{deg}_{G}(u)}{2}-\alpha-\frac{1}{2}\right) \\
& \geq \alpha\left(\frac{1}{2} \delta n-\alpha-\frac{1}{2}\right) .
\end{aligned}
$$

Since there are $\operatorname{cut}_{r+1}(G)-1$ neutral edges in $G$, we have

$$
\alpha\left(\frac{1}{2} \delta n-\alpha-\frac{1}{2}\right) \leq \operatorname{cut}_{r+1}(G)-1 \leq(n-r-1)(r+1)-1 .
$$

One can check that if $N$ is large enough, then the inequality $\frac{1}{2} \delta n \alpha-\alpha^{2}-\frac{1}{2} \alpha \leq(n-$ $r-1)(r+1)-1$ implies that we have either $r+1 \leq \alpha \leq \frac{3(r+1)}{\delta}$ or $\frac{\delta}{2} n-o(n) \leq \alpha \leq \frac{n}{2}$. Similarly, we have either $r+1 \leq \beta \leq \frac{3(r+1)}{\delta}$ or $\frac{\delta}{2} n-o(n) \leq \beta \leq \frac{n}{2}$. These observations lead us to investigate the following two cases. In what follows, we will show that these cases do not hold.

Case I). $\frac{\delta}{2} n-o(n) \leq \alpha \leq \frac{n}{2}$ or $\frac{\delta}{2} n-o(n) \leq \beta \leq \frac{n}{2}$.

Because of similarity, we just discuss the case $\frac{\delta}{2} n-o(n) \leq \alpha \leq \frac{n}{2}$. For a contradiction, suppose that $\frac{\delta}{2} n-o(n) \leq \alpha \leq \frac{n}{2}$. Consider the bipartite graph $H_{R}$ and note that

$$
\left|E\left(H_{R}\right)\right| \geq \alpha(\delta n-\alpha+1) \geq \alpha(\delta n-\alpha) \geq\left(\frac{2 \delta-1}{4}\right) n^{2}-o\left(n^{2}\right)
$$

provided that $N$ is large enough. To see this, note that the function $f(\alpha)=\alpha(\delta n-\alpha)$ for $\alpha \in\left[\frac{\delta}{2} n-o(n), \frac{n}{2}\right]$, takes its minimum in $\frac{\delta}{2} n-o(n)$ or $\frac{n}{2}$. Comparing two values $f\left(\frac{\delta}{2} n-o(n)\right)$ and $f\left(\frac{n}{2}\right)$, we have the aforementioned inequality. Since, there is no red edge between two parts $U_{R}$ and $V_{R}$, and moreover, there are at most $(n-r-1)(r+1)-1=o\left(n^{2}\right)$ neutral edges between them, one can conclude that there are at least $\left(\frac{2 \delta-1}{4}\right) n^{2}-o\left(n^{2}\right)$ blue edges in $E\left(H_{R}\right)$.

We call an edge of $G$ unusual, if it is either a neutral edge of $G$ or a blue edge in $E(G) \backslash E\left(H_{R}\right)$. We remind that two 3-blocks are called consecutive if they are traversed 
consecutively in the Eulerian tour of $G-E(M)$ used for introducing the ordering $\sigma$. In other words, if two 3 -blocks are consecutive, then there is no other edge between the edges of them in the ordering $\sigma$. Set $q$ to be the number of 3-blocks. For any $i \in[n]$, let $q_{i}$ be the number of 3 -blocks which are traversed in the $i$-th stage. One can check that amongst the edges of any two consecutive 3-blocks, there exists at least one unusual edge. Therefore, there are at least $\frac{q_{i}-1}{2}$ unusual edges amongst the edges of these $q_{i}$ 3 -blocks. Hence, we have at least $\frac{q-n}{2}$ unusual edges in $G$. In view of Equality 3 and the aforementioned discussion, the number of unusual edges is at least

$$
\frac{1}{2}\left(\frac{|E(G)|}{3 n(n-2)}\left(4|E(G)|-n^{2}\right)-o\left(n^{2}\right)\right)-\frac{n}{2}=\frac{|E(G)|}{6 n(n-2)}\left(4|E(G)|-n^{2}\right)-o\left(n^{2}\right)
$$

Also, note that there are at most $(n-r-1)(r+1)-1=o\left(n^{2}\right)$ neutral edges in $E(G)$. Accordingly, the number of blue edges in $G$ is

$$
\left|E\left(G^{B}\right) \backslash E\left(H_{R}\right)\right|+\left|E\left(G^{B}\right) \cap E\left(H_{R}\right)\right| \geq \frac{|E(G)|}{6 n^{2}}\left(4|E(G)|-n^{2}\right)+\frac{2 \delta-1}{4} n^{2}-o\left(n^{2}\right) .
$$

Since $\delta>\frac{5}{6}$, one can see that there is a $\mu=\mu(\delta)>0$ for which

$$
\frac{|E(G)|}{6 n^{2}}\left(4|E(G)|-n^{2}\right)+\frac{2 \delta-1}{4} n^{2}>(1+\mu) \frac{|E(G)|}{2} .
$$

To see this, set $e=|E(G)| \in\left(\frac{5 n^{2}}{12}, \frac{n^{2}}{2}\right]$ and note that the function

$$
g(e)=\frac{e}{6 n^{2}}\left(4 e-n^{2}\right)+\frac{2 \delta-1}{4} n^{2}-(1+\mu) \frac{e}{2}
$$

is decreasing for $e \in\left(\frac{5 n^{2}}{12}, \frac{n^{2}}{2}\right]$. Consequently, it takes its minimum in $e=\frac{n^{2}}{2}$ which is

$$
g\left(\frac{n^{2}}{2}\right)=\left(2 \delta-\frac{5}{3}-\mu\right) \frac{n^{2}}{4} .
$$

Choosing $\mu$ sufficiently small, for instance $\mu=\delta-\frac{5}{6}$, we clearly have $g\left(\frac{n^{2}}{2}\right)>0$. Therefore, since $\frac{\mu}{2}|E(G)|=O\left(n^{2}\right)$, for large enough $N$, we have

$$
\left|E\left(G^{B}\right) \backslash E\left(H_{R}\right)\right|+\left|E\left(G^{B}\right) \cap E\left(H_{R}\right)\right|>\frac{|E(G)|}{2}+1,
$$

contradicting the fact that the number of blue edges in $G$ is at most $\frac{|E(G)|}{2}+1$. Similarly, if $\frac{\delta}{2} n-o(n) \leq \beta \leq \frac{n}{2}$, then we get a contradiction.

Case II). $r+1 \leq \alpha \leq \frac{3(r+1)}{\delta}$ or $r+1 \leq \beta \leq \frac{3(r+1)}{\delta}$. 
Because of similarity, we just discuss the case $r+1 \leq \alpha \leq \frac{3(r+1)}{\delta}$. For a contradiction, suppose that $r+1 \leq \alpha \leq \frac{3(r+1)}{\delta}$. Note that for any vertex $x \in U_{R} \cap U_{B}$, all edges between $x$ and the vertices in $V(G) \backslash\left(U_{R} \cup U_{B}\right)$ are neutral, i.e., each edge in $E_{G}\left(U_{R} \cap U_{B}, V(G) \backslash\right.$ $\left.\left(U_{R} \cup U_{B}\right)\right)$ is neutral. Clearly, since $r=o(n)$, if $\left|U_{R} \cap U_{B}\right| \geq \frac{2(r+1)}{\delta}$ and $N$ is sufficiently large, then the number of neutral edges in $G$ is at least

$$
\left|U_{R} \cap U_{B}\right|\left(\delta n-\left|U_{R} \cup U_{B}\right|\right) \geq \frac{2(r+1)}{\delta}\left(\delta n-\frac{6(r+1)}{\delta}\right)>(r+1)(n-r-1)
$$

which is impossible. Now, suppose $\left|U_{R} \cap U_{B}\right| \leq \frac{2(r+1)}{\delta}$.

Consider a vertex $v_{i} \in U_{R} \backslash U_{B}$ (if there is such a vertex). Since all 4-blocks are chosen from a monogamous packing, the number of 4-blocks in $H_{i}$ having some vertex in $U_{R} \cup U_{B} \backslash\left\{v_{i}\right\}$ is at most $\left|U_{R} \cup U_{B}\right|-1 \leq \frac{6(r+1)}{\delta}-1$.

Also, it is clear that any 4 -block in $H_{i}$ which has no red edge incident with $v_{i}$ contains at most one red edge. Note that if a 4-block in $H_{i}$ has a red edge incident with $v_{i}$, then it contains some vertex in $U_{R} \cup U_{B} \backslash\left\{v_{i}\right\}$. Suppose that there are $\gamma$ consecutive 4-blocks of $H_{i}$ (with respect to the ordering $\sigma$ ) such that each of them has no vertex in $U_{R} \cup U_{B} \backslash\left\{v_{i}\right\}$. Clearly, these 4-blocks have no red edge incident with $v_{i}$. Consequently, they have at most $\gamma$ red edges. Also, since the edges of these 4-blocks are consecutive in $\sigma$, they contain at most $\gamma+1$ blue edges. This implies that there are at least $4 \gamma-(2 \gamma+1)=2 \gamma-1$ neutral edges amongst the edges of these 4-blocks. Since 4-blocks form a monogamous $C_{4}$-packing of $G$, the number of 4 -blocks in $H_{i}$ containing some vertex of $\left(U_{R} \cup U_{B}\right) \backslash\left\{v_{i}\right\}$ is at most $\left|U_{R} \cup U_{B}\right|-1$. This observation implies that

$$
\left|\operatorname{NEU}\left(H_{i}-\left(U_{R} \cup U_{B} \backslash\left\{v_{i}\right\}\right)\right)\right| \geq 2 l-3\left|U_{R} \cup U_{B}\right|+2 .
$$

Moreover, in view of the definition of $\sigma$, there are at least $\frac{\operatorname{deg}_{G}\left(v_{i}\right)-3}{2}$ edge-disjoint $\left(\sigma, v_{i}\right)$-consecutive paths in $G$. Amongst these $\left(\sigma, v_{i}\right)$-consecutive paths, at most $l+1$ (resp. $\left|U_{R} \cup U_{B}\right|-1$ ) number of them have nonempty intersection with $E\left(H_{i}\right)$ (resp. $\left.E\left(G\left[U_{R} \cup U_{B}\right]\right)\right)$. If we set $E_{v_{i}}^{R}=E\left(H_{i}\right) \cup E\left(G\left[U_{R} \cup U_{B}\right]\right)$, then in view of Lemma 1 , the number of neutral edges incident with $v_{i}$ which are not in $E\left(H_{i}\right) \cup E\left(G\left[U_{R} \cup U_{B}\right]\right)$, i.e., $\left|\operatorname{NEU}\left(v_{i}, G-\left(E\left(H_{i}\right) \cup E\left(G\left[U_{R} \cup U_{B}\right]\right)\right)\right)\right|$, is at least

$$
\frac{\operatorname{deg}_{G}\left(v_{i}\right)-3}{2}-(l+1)-\left(\left|U_{R} \cup U_{B}\right|-1\right)=\frac{1}{2} \operatorname{deg}_{G}\left(v_{i}\right)-l-\left|U_{R} \cup U_{B}\right|-\frac{3}{2}
$$

Similarly, with the same argument, we can obtain the same assertions for any vertex $v_{j} \in U_{B} \backslash U_{R}$. Hence, in view of Inequality 7 and since $l=\left\lceil\frac{26 r+26}{\delta}\right\rceil$, for any vertex $v_{i} \in\left(U_{R} \backslash U_{B}\right) \cup\left(U_{B} \backslash U_{R}\right)=U_{R} \cup U_{B} \backslash U_{R} \cap U_{B}$, we have 


$$
\begin{aligned}
& \left|\operatorname{NEU}\left(v_{i}, G-\left(E\left(H_{i}\right) \cup E\left(G\left[U_{R} \cup U_{B}\right]\right)\right)\right)\right|+\left|\operatorname{NEU}\left(H_{i}-\left(U_{R} \cup U_{B} \backslash\left\{v_{i}\right\}\right)\right)\right| \\
\geq & \frac{1}{2} \operatorname{deg}_{G}\left(v_{i}\right)-l-\left|U_{R} \cup U_{B}\right|-\frac{3}{2}+2 l-3\left|U_{R} \cup U_{B}\right|+2 \\
= & \frac{\operatorname{deg}\left(v_{i}\right)}{2}+l-4\left|U_{R} \cup U_{B}\right|+\frac{1}{2} \\
> & \frac{\operatorname{deg}\left(v_{i}\right)}{2}+\frac{2(r+1)}{\delta} .
\end{aligned}
$$

If $u, u^{\prime} \in U_{R} \cap U_{B}$ and $v_{i}, v_{j} \in\left(U_{R} \cup U_{B}\right) \backslash\left(U_{R} \cap U_{B}\right)$, such that $u \neq u^{\prime}$ and $v_{i} \neq v_{j}$, then

$$
\begin{gathered}
\operatorname{NEU}\left(u, G-\left(U_{R} \cup U_{B} \backslash\{u\}\right)\right) \cap \operatorname{NEU}\left(u^{\prime}, G-\left(U_{R} \cup U_{B} \backslash\left\{u^{\prime}\right\}\right)\right)=\varnothing, \\
\operatorname{NEU}\left(u, G-\left(U_{R} \cup U_{B} \backslash\{u\}\right)\right) \cap \operatorname{NEU}\left(v_{i}, G-\left(E\left(H_{i}\right) \cup E\left(G\left[U_{R} \cup U_{B}\right]\right)\right)\right)=\varnothing, \\
\operatorname{NEU}\left(u, G-\left(U_{R} \cup U_{B} \backslash\{u\}\right)\right) \cap \operatorname{NEU}\left(H_{i}-\left(U_{R} \cup U_{B} \backslash\left\{v_{i}\right\}\right)\right)=\varnothing,
\end{gathered}
$$$$
\operatorname{NEU}\left(v_{i}, G-\left(E\left(H_{i}\right) \cup E\left(G\left[U_{R} \cup U_{B}\right]\right)\right)\right) \cap \operatorname{NEU}\left(v_{j}, G-\left(E\left(H_{j}\right) \cup E\left(G\left[U_{R} \cup U_{B}\right]\right)\right)\right)=\varnothing,
$$$$
\operatorname{NEU}\left(H_{i}-\left(U_{R} \cup U_{B} \backslash\left\{v_{i}\right\}\right)\right) \cap \operatorname{NEU}\left(v_{j}, G-\left(E\left(H_{j}\right) \cup E\left(G\left[U_{R} \cup U_{B}\right]\right)\right)\right)=\varnothing,
$$

and

$$
\operatorname{NEU}\left(H_{i}-\left(U_{R} \cup U_{B} \backslash\left\{v_{i}\right\}\right)\right) \cap \operatorname{NEU}\left(H_{j}-\left(U_{R} \cup U_{B} \backslash\left\{v_{j}\right\}\right)\right)=\varnothing .
$$

In what follows, by using the preceding equations, we shall present a lower bound for the number of neutral edges in $G$, leading us to a contradiction. Before this, we remind that all edges between vertices in $U_{R} \cap U_{B}$ and the vertices in $V(G) \backslash\left(U_{R} \cup U_{B}\right)$ are neutral.

$$
\begin{aligned}
|\mathrm{NEU}(G)| & \geq \sum_{u \in U_{R} \cap U_{B}}\left|\operatorname{NEU}\left(u, G-\left(U_{R} \cup U_{B} \backslash\{u\}\right)\right)\right| \\
& +\sum_{v_{i} \in\left(U_{R} \cup U_{B}\right) \backslash U_{R} \cap U_{B}}\left|\operatorname{NEU}\left(v_{i}, G-\left(E\left(H_{i}\right) \cup E\left(G\left[U_{R} \cup U_{B}\right]\right)\right)\right)\right| \\
& +\sum_{v_{i} \in\left(U_{R} \cup U_{B}\right) \backslash U_{R} \cap U_{B}}\left|\operatorname{NEU}\left(H_{i}-\left(U_{R} \cup U_{B} \backslash\left\{v_{i}\right\}\right)\right)\right| \\
& \geq\left|E_{G}\left(U_{R} \cap U_{B}, V(G) \backslash\left(U_{R} \cup U_{B}\right)\right)\right|+ \\
& +\sum_{v \in\left(U_{R} \cup U_{B}\right) \backslash\left(U_{R} \cap U_{B}\right)}\left(\frac{\operatorname{deg}_{G}(v)}{2}+\frac{2(r+1)}{\delta}\right) .
\end{aligned}
$$

\section{Define}




$$
\begin{aligned}
J & =\sum_{v \in\left(U_{R} \cup U_{B}\right) \backslash\left(U_{R} \cap U_{B}\right)}\left(\frac{\operatorname{deg}_{G}(v)}{2}+\frac{2(r+1)}{\delta}\right) \\
& =\frac{2(r+1)}{\delta}\left|\left(U_{R} \cup U_{B}\right) \backslash\left(U_{R} \cap U_{B}\right)\right| \quad+\sum_{v \in\left(U_{R} \cup U_{B}\right) \backslash\left(U_{R} \cap U_{B}\right)} \frac{\operatorname{deg}_{G}(v)}{2} .
\end{aligned}
$$

Let $\left(U_{R} \cup U_{B}\right) \backslash\left(U_{R} \cap U_{B}\right)=\left\{u_{1}, u_{2}, \ldots, u_{d}\right\}$, where $\operatorname{deg}_{G}\left(u_{1}\right) \leq \operatorname{deg}_{G}\left(u_{2}\right) \leq \cdots \leq$ $\operatorname{deg}_{G}\left(u_{d}\right)$. Note that $d \geq 2 r+2-2\left|U_{R} \cap U_{B}\right|$. Set

$$
d^{\prime}=\max \left\{0, r+1-\left|U_{R} \cap U_{B}\right|\right\}, Y=\left\{u_{1}, u_{2}, \ldots, u_{d^{\prime}}\right\}, \text { and } Z=Y \cup\left(U_{R} \cap U_{B}\right),
$$

where $Y=\varnothing$ if $d^{\prime}=0$. Note that $r+1 \leq|Z| \leq \frac{6(r+1)}{\delta} \leq n-r-1$. Consequently, we have

$$
\begin{aligned}
|\mathrm{NEU}(G)| & \geq\left|E_{G}\left(U_{R} \cap U_{B}, V(G) \backslash\left(U_{R} \cup U_{B}\right)\right)\right|+J \\
& \geq\left|E_{G}\left(U_{R} \cap U_{B}, V(G) \backslash\left(U_{R} \cup U_{B}\right)\right)\right|+\frac{2(r+1)}{\delta}\left|\left(U_{R} \cup U_{B}\right) \backslash\left(U_{R} \cap U_{B}\right)\right| \\
& +\sum_{u \in\left(U_{R} \cup U_{B}\right) \backslash\left(U_{R} \cap U_{B}\right)} \frac{\operatorname{deg}_{G}(u)}{2} .
\end{aligned}
$$

Clearly, since $\left|U_{R} \cap U_{B}\right| \leq \frac{2(r+1)}{\delta}$, we have

$$
\begin{aligned}
& \left|E_{G}\left(U_{R} \cap U_{B}, V(G) \backslash\left(U_{R} \cup U_{B}\right)\right)\right|+\frac{2(r+1)}{\delta}\left|\left(U_{R} \cup U_{B}\right) \backslash\left(U_{R} \cap U_{B}\right)\right| \\
& \quad \geq\left|E_{G}\left(U_{R} \cap U_{B}, V(G) \backslash\left(U_{R} \cap U_{B}\right)\right)\right| .
\end{aligned}
$$

Accordingly, since $2 d^{\prime} \leq d$, we have

$$
\begin{aligned}
|\mathrm{NEU}(G)| & \geq\left|E_{G}\left(U_{R} \cap U_{B}, V(G) \backslash\left(U_{R} \cap U_{B}\right)\right)\right|+\sum_{u \in\left(U_{R} \cup U_{B}\right) \backslash\left(U_{R} \cap U_{B}\right)} \frac{\operatorname{deg}_{G}(u)}{2} \\
& \geq \mid E_{G}\left(U_{R} \cap U_{B}, V(G) \backslash\left(U_{R} \cap U_{B}\right) \mid+\sum_{u \in Y} \operatorname{deg}_{G}(u)\right. \\
& \geq\left|E_{G}(Z, V(G) \backslash Z)\right| \\
& \geq \operatorname{cut}_{r+1}(G),
\end{aligned}
$$

a contradiction.

Clearly the aforementioned claim implies the desired assertion of Part (II) which completes the proof of lemma. 


\subsection{Proof of Theorem 2}

To prove Theorem 2, we use the same approach as in the proof of Theorem 1 with $r=0$. This assumption makes the computation easier.

Proof of Theorem 2. Let $G$ be a graph with $n \geq 4716$ vertices and $\delta(G) \geq \delta n$. To prove the assertion, we follow the proof of Theorem 1 with $r=0$. Note that $l=\left\lceil\frac{26}{\delta}\right\rceil \leq 32$. In the definition of $\sigma$, we need the inequality $n \geq 36(4 l+3)$ which is valid for $n \geq 4716$. Also, for using Dirac's theorem, the inequality $\delta n-8 l-6 \geq \frac{n}{2}+2$ should be satisfied which is due to the assumption $n \geq 4716$. Continuing the proof of Theorem 1 , we meet the following inequalities:

$$
\frac{1}{2} \delta n \alpha-\alpha^{2}-\frac{1}{2} \alpha \leq n-2 \text { and } \frac{1}{2} \delta n \beta-\beta^{2}-\frac{1}{2} \beta \leq n-2 .
$$

For $n \geq 27$, these inequalities lead us to the following cases:

(I) Either $1 \leq \alpha \leq \frac{3}{\delta}$ or $\frac{\delta}{2} n-7 \leq \alpha \leq \frac{n}{2}$.

(II) Either $1 \leq \beta \leq \frac{3}{\delta}$ or $\frac{\delta}{2} n-7 \leq \beta \leq \frac{n}{2}$.

Same as in the proof of Theorem 1, we distinguish two different cases, each of them leads us to a contradiction.

Case I). $\frac{\delta}{2} n-7 \leq \alpha \leq \frac{n}{2}$ or $\frac{\delta}{2} n-7 \leq \beta \leq \frac{n}{2}$.

We have at least $\left(\frac{2 \delta-1}{4}\right) n^{2}-49$ edges in $E\left(H_{R}\right)$. Also, the number of 3 -blocks is at least

$$
\frac{e-y}{3 n^{2}}\left(4 e-4 y-n^{2}\right) \frac{(n-3)(n-4)}{(n-1)(n-2)} \geq \frac{e-y}{3 n^{2}}\left(4 e-4 y-n^{2}\right)\left(1-\frac{5}{n}\right),
$$

where $e=|E(G)|$ and $y=|E(L)|$. Since $y \leq(4 l+4) n \leq 132 n$, we have at least

$$
\begin{aligned}
\frac{e-y}{3 n^{2}}\left(4 e-4 y-n^{2}\right)\left(1-\frac{5}{n}\right) & \geq \frac{e-132 n}{3 n^{2}}\left(4 e-528 n-n^{2}\right)\left(1-\frac{5}{n}\right) \\
& \geq \frac{e}{3 n^{2}}\left(4 e-n^{2}\right)-\frac{797}{6} n
\end{aligned}
$$

3 -blocks. Hence, we have at least $\frac{e}{6 n^{2}}\left(4 e-n^{2}\right)-\frac{797}{12} n-\frac{n}{2}$ unusual edges. Note that since there is no red edge in $E\left(H_{R}\right)$ and there is at most $(n-2)$ neutral edges in $G$, we have at least

$$
\left(\frac{2 \delta-1}{4}\right) n^{2}-49-(n-2)+\frac{e}{6 n^{2}}\left(4 e-n^{2}\right)-\frac{803}{12} n-(n-2)
$$

blue edges in $G$. We claim that for $n \geq \frac{828}{6 \delta-5}$,

$$
\left(\frac{2 \delta-1}{4}\right) n^{2}+\frac{e}{6 n^{2}}\left(4 e-n^{2}\right)-\frac{827}{12} n-45>\frac{e}{2}+1
$$


which is a contradiction. To see this, note that the function

$$
g(e)=\left(\frac{2 \delta-1}{4}\right) n^{2}+\frac{2}{3 n^{2}} e^{2}-\frac{2}{3} e-\frac{827}{12} n-46
$$

takes its minimum in $e=\frac{n^{2}}{2}$ which is

$$
g\left(\frac{n^{2}}{2}\right)=\left(\frac{6 \delta-5}{12}\right) n^{2}-\frac{827}{12} n-46>0
$$

provided that $n \geq \frac{828}{6 \delta-5}$. The rest of the proof runs as before.

\section{Complexity of finding the $t$-th cut number}

In Theorem 1, it is proved that the chromatic number of $\operatorname{KG}\left(G, \mathcal{T}_{n-r}\right)$ is equal to $\operatorname{cut}_{r+1}(G)$ provided that $G$ satisfies some conditions. This motivates us to consider the problem of finding the $t$-th cut number of a given graph $G$. Note that, in addition to being motivated by Theorem 1, this problem is interesting for its own sake. In this regard, we consider the following problem. Let $t=t(n)$ be a positive function such that $t(n) \leq \frac{n}{2}$ for each $n \in \mathbb{N}$. For a given graph $G$ with $n$ vertices, what is the value of

$$
\operatorname{cut}_{t}(G)=\min \{|E(S, V(G) \backslash S)|: S \subseteq V(G), \min \{|S|,|V(G)|-|S|\} \geq t\} ?
$$

Hereafter, by Min $t$-Cut problem, we refer to this problem. We remind that for $t \equiv 1$, $\operatorname{cut}_{1}(G)=\operatorname{cut}(G)$ is called the cut number of $G$. Also, we prefer to use Min-Cut problem instead of Min 1-Cut problem.

The famous max-flow min-cut theorem says that the maximum network flow from the source $s$ to the sink $t$ in a network $N$ equals to the minimum weighted cut-number of $N$, denoted $\operatorname{cut}(N)$ which is the smallest total weight of the edges which if removed would disconnect the source from the sink (there is no directed path from $s$ to $t$ ). Hao and Orlin 1992 [8] showed how to use the maximum flow algorithm by Goldberg and Tarjan 1988 [6] in order to find the minimum weighted cut-number of a network $N$ in polynomial time of $|V(N)|$. There are other polynomial time methods for finding the minimum weighted cut-number in $N$, notably the Edmonds-Karp algorithm [4]. Edmonds-Karp algorithm ensures that we can find the maximum flow in any network $N$ with $n$ vertices and $m$ edges in $O\left(\mathrm{~nm}^{2}\right)$ time. In what follows, with the similar idea as Goldberg and Tarjan used, we prove that the problem of finding $\operatorname{cut}_{t}(G)$ can be solved in a polynomial time of $|V(G)|$, where $t$ is a constant function. For a given graph $G$ with $|V(G)|=n$, define $\vec{G}$ to be a directed graph with the same vertex set as $G$ and for any edge $e=u v \in E(G)$, put two edges $(u, v)$ and $(v, u)$ in $\vec{G}$. Also, for each edge in $\vec{G}$, define its capacity to be 1 . Now, let $(A, B)$ be a pair of disjoint $t$-subsets of $V(G)$. Define $N_{(A, B)}$ to be a network with vertex set $V(G) \cup\{s, t\}$ and edge set 


$$
E\left(N_{(A, B)}\right)=E(\vec{G}) \cup\{(s, a): a \in A\} \cup\{(b, t): b \in B\}
$$

Also, set the capacity of each edge incident with $s$ or $t$ to be infinite. Note that the max-flow min-cut theorem and Edmonds-Karp algorithm imply that we can find the minimum cut of $N_{(A, B)}$ in $O\left(n^{5}\right)$ time. On the other hand, one can simply see that

$$
\operatorname{cut}_{t}(G)=\min _{(A, B)} \operatorname{cut}\left(N_{(A, B)}\right)
$$

where the minimum is taken over all pairs $(A, B)$ of disjoint $t$-subsets of $V(G)$. Since $t$ is a constant and the number of such pairs $(A, B)$ is $\left(\begin{array}{c}n \\ t\end{array}\right)\left(\begin{array}{c}n-t \\ t\end{array}\right)=O\left(n^{2 t}\right)$, the minimum can be found in polynomial time. Hence, we can find $\operatorname{cut}_{t}(G)$ in polynomial time of $n$. However, it is proved that the complexity of finding the $t$-th cut number when $t$ is a part of the input is NP-hard. In this regard, the Min $\left\lfloor\frac{n}{2}\right\rfloor$-Cut problem, known as the minimum bisection problem in the literature, is proved to be an $N P$-hard problem by Garey, Johnson, and Stockmeyer [5]. Bui and Jones [3] showed that allowing the two sets to be unequal does not make the problem easier while we have a lower bound on the size of smaller set. To be more specific, let $b$ be a constant, where $0<b \leq \frac{1}{2}$. In a graph $G$, a cut $(S, T)$ is called $b$-balanced if $\min \{|S|,|T|\} \geq b|V(G)|$. The minimum number of edges in the $b$-balanced cuts is called the b-balanced cut number of $G$. Bui and Jones [3] proved that the problem of finding the $b$-balanced cut number is an NP-hard problem.

However, it would be desirable for us to know the complexity of Min $t$-Cut problem whenever the function $t(n)$ and the given graphs satisfy the conditions of Theorem 1 . In the rest of this section, we focus on investigating this problem. By the next theorem, we shall prove that the Min $t$-Cut problem still remains NP-hard even if the given graphs are satisfying the conditions of Theorem 1 and the function $t(n)$ is too small with respect to $n$. Let us first introduce a famous NP-complete problem in graphs, known as Max-Cut problem which has a key role in the proof of the next theorem. Finding the maximum size of a cut in a graph $\mathrm{G}$ is known as Max-Cut problem. Also, the decision version of the Max-Cut problem is the following problem: Given a graph $G$ and an integer $k \in \mathbb{N}$, is there a cut of size at least $k$ in $G$ ? It is proved to be an NP-complete problem by Garey, Johnson, and Stockmeyer [5]. Therefore, the above computational version has to be an NP-hard problem. One should notice that the proof of the following theorem is similar to a result in [5].

Theorem 3. Let $k \geq 2$ be an arbitrary fixed positive integer and $n$ be a positive integer. The Min $n^{\frac{1}{k}}$-Cut problem is an NP-hard problem even for the family of graphs with $n$ vertices satisfying the hypotheses of Theorem 1.

Proof. To prove the theorem, we introduce a reduction from Max-Cut problem to Min $n^{\frac{1}{k}}$-Cut problem. To this end, let $G$ be a graph with $m$ vertices which is an input for Max-Cut problem. As an input for Min $n^{\frac{1}{k}}$-Cut problem, define $H=\bar{G} \vee K_{q}$, where $q=m^{k}-m$ and $V\left(K_{q}\right)=\{1, \ldots, q\}$. Since $H$ has $n=m^{k}$ vertices and $\delta(H) \geq n-n^{\frac{1}{k}}$, it satisfies the conditions of Theorem 1 provided that $n$ is large enough. 
Let $\left(U_{0}, V_{0}\right)$ be a partition of $V(G)$ for which we have

$$
\left|E_{G}\left(U_{0}, V_{0}\right)\right|=\max \left\{\left|E_{G}(U, V)\right|:(U, V) \text { is a cut of } G\right\} \text {. }
$$

Set $S=U_{0} \cup\left\{1, \ldots, n^{\frac{1}{k}}-\left|U_{0}\right|\right\}$ and $T=V(H) \backslash S$. Note that $|S|=n^{\frac{1}{k}}$ and $|T|=$ $n-n^{\frac{1}{k}} \geq n^{\frac{1}{k}}$ for $n \geq 4$. Therefore, $(S, T)$ is an $n^{\frac{1}{k}}$-cut of $H$. Moreover, we clearly have

$$
\operatorname{cut}_{n^{\frac{1}{k}}}(H) \leq\left|E_{H}(S, T)\right|=n^{\frac{1}{k}}\left(n-n^{\frac{1}{k}}\right)-\left|E_{G}\left(U_{0}, V_{0}\right)\right| .
$$

Conversely, let $(S, T)$ be an $n^{\frac{1}{k}}$-cut of $H$ for which we have $\left|E_{H}(S, T)\right|=\operatorname{cut}_{n^{\frac{1}{k}}}(H)$. Define $U=S \cap V(G)$ and $V=V(G) \backslash U$. Clearly, since $\min \{|S|,|T|\} \geq n^{\frac{1}{k}}$, we have

$$
\left|E_{G}\left(U_{0}, V_{0}\right)\right| \geq\left|E_{G}[U, V]\right|=|S||T|-\left|E_{H}[S, T]\right| \geq n^{\frac{1}{k}}\left(n-n^{\frac{1}{k}}\right)-\operatorname{cut}_{n^{\frac{1}{k}}}(H) .
$$

Therefore, in view of Equations (10) and (11), we have $\left|E\left(U_{0}, V_{0}\right)\right|=n^{\frac{1}{k}}\left(n-n^{\frac{1}{k}}\right)-$ cut $_{n^{\frac{1}{k}}}(H)$. Consequently, solving Min $n^{\frac{1}{k}}$-Cut problem, even for graphs satisfying the conditions of Theorem 1, results polynomially in solving Max-Cut problem which is an NP-hard problem, completing the proof.

In view of the previous theorem, for any fixed number $\epsilon \in(0,1)$, Max-Cut problem and Min $t$-Cut problem with $t \geq n^{\epsilon}$ are both NP-hard while, as an interesting comparison, Min $t$-Cut problem with constant $t$ is solvable in polynomial time (see the discussion before Theorem 3). As a result of Theorem 1 and the preceding observation, one should note that, for the family of graphs $G$ satisfying the conditions of Theorem 1, the problem of computing the chromatic number of tree Kneser graphs $\operatorname{KG}\left(G, \mathcal{T}_{t}\right)$ is polynomially solvable if $t$ is fixed, while it is an NP-hard problem provided that $t=n^{\frac{1}{k}}$ for some fixed integer $k \geq 2$.

It is worth mentioning that by a similar approach as in the proof of Theorem 3, we can prove a theorem similar to Theorem 3 for the decision version of the Min $t$-Cut problem.

Remark. There is an unpublished version of the present paper available on arXive (arXive:1407.8035v2) containing a generalization of Theorem 1. This generalization can be somehow considered as a colored version of Theorem 1 which determines the chromatic number of several induced subgraphs of $\operatorname{KG}\left(G, \mathcal{T}_{n-r}\right)$. This generalization is too technical and not easy to follow. We did not put it in the journal version of the paper since we have preferred clarity to generality and also to keep the paper of broad enough interest.

\section{Acknowledgments}

The research of Hossein Hajiabolhassan was supported by ERC advanced grant GRACOL. Furthermore, part of this work was done during a visit of Hossein Hajiabolhassan to the Mittag-Leffler Institute (Djursholm, Sweden). We are grateful to Dr. Roya 
Abyazisani and Professor Carsten Thomassen for their fruitful discussions and comments. Also, we gratefully acknowledge the anonymous referee for the constructive comments that helped us to improve the presentation of the paper. Moreover, the author would like to thank Skype for sponsoring their endless conversations in two countries.

\section{References}

[1] M. Alishahi, H. Hajiabolhassan, On the chromatic number of general Kneser hypergraphs, J. Combin. Theory Ser. B 115 (2015) 186-209.

[2] M. Alishahi, H. Hajiabolhassan, Chromatic number via Turán number, Discrete Math. 340 (10) (2017) 2366-2377.

[3] T.N. Bui, C. Jones, Finding good approximate vertex and edge partitions is NP-hard, Inform. Process. Lett. 42 (3) (1992) 153-159.

[4] J. Edmonds, R.M. Karp, Theoretical improvements in algorithmic efficiency for network flow problems, J. ACM 19 (1972) 4.

[5] M.R. Garey, D.S. Johnson, L. Stockmeyer, Some simplified NP-complete graph problems, Theoret. Comput. Sci. 1 (1976).

[6] A.V. Goldberg, R.E. Tarjan, A new approach to the maximum-flow problem, J. ACM 35 (1988) 10.

[7] A. Hajnal, E. Szemerédi, Proof of a conjecture of P. Erdős, in: Combinatorial Theory and Its Applications, II, Proc. Colloq., Balatonfüred, 1969, North-Holland, Amsterdam, 1970, pp. 601-623.

[8] J.X. Hao, J.B. Orlin, A faster algorithm for finding the minimum cut in a directed graph, J. Algorithms 17 (1994).

[9] N.H. Hoi, On the Problems of Edge Disjoint Cliques in Graphs, PhD thesis, Eötvös Loránd University, Mathematics Department, 2005.

[10] M. Kneser, Ein Satz über abelsche Gruppen mit Anwendungen auf die Geometrie der Zahlen, Math. Z. 61 (1955) 429-434.

[11] S. Kundu, Bounds on the number of disjoint spanning trees, J. Combin. Theory Ser. B 17 (1974) 199-203.

[12] C.C. Lindner, A. Rosa, Monogamous decompositions of complete bipartite graphs, symmetric Hsquares, and self-orthogonal 1-factorizations, Australas. J. Combin. 20 (1999) 251-256.

[13] L. Lovász, Kneser's conjecture, chromatic number, and homotopy, J. Combin. Theory Ser. A 25 (3) (1978) 319-324.

[14] C.St.J.A. Nash-Williams, Edge-disjoint spanning trees of finite graphs, J. Lond. Math. Soc. s1-36 (1961) 01.

[15] W.T. Tutte, On the problem of decomposing a graph into n connected factors, J. Lond. Math. Soc. s1-36 (1961) 01. 